DOI: http://dx.doi.org/10.12957/demetra.2014.11608

\title{
Produção científica sobre alimentos funcionais: uma análise das publicações brasileiras entre 2007 e 2013
}

\section{Scientific production on functional food: an analysis of Brazilian publications between 2007 and 2013}

Ítalo de Paula Casemiro'

Paula Ramos'

' Centro de Ciências da Saúde. Universidade Federal do Rio de Janeiro. Cidade Universitária, Rio de Janeiro-RJ, Brasil.

Correspondência / Correspondence Ítalo de Paula Casemiro

E-mail: itcasemiro@hotmail.com

\section{Resumo}

A produção científica brasileira sobre alimentos funcionais corresponde ao objeto de estudo do presente artigo. Trata-se de levantamento, por meio de técnicas bibliométricas, de artigos publicados sobre a temática em questão no período entre 2007 a 2013, disponibilizado no Portal de Periódicos da Capes. Dos 164 artigos em língua portuguesa encontrados no referido portal, 22 foram selecionados para análise. Sobre o tema em questão, foram encontrados artigos de oito periódicos, sendo que dois concentravam 63,63\% da produção: Alimentos e Nutrição (36,36\%) e Ciência e Tecnologia de Alimentos (27,27\%). Os temas abordados paralelamente foram antioxidantes, ácidos graxos, prebióticos e probióticos. A UNICAMP foi a instituição com a maior produção de artigos analisados, representando 13,64\% da amostra. Quanto à abordagem de método, verifica-se predominância do método quantitativo $(54,54 \%)$, que pode ser justificado pelo emprego de avaliações sensoriais e físico-químicas, instrumentos comumente utilizados para análises quantitativas encontrados em 45,45\% dos artigos pesquisados. A maioria dos autores possui formação em Nutrição e estão vinculados às universidades. A pesquisa foi exploratória com o uso da análise documental, no primeiro momento, e descritiva, com método quantitativo, no segundo momento. A análise considerou o número de artigos, instituições de ensino superior, temas, metodologia, instrumentos de coleta de dados e área de formação dos autores. Os trabalhos publicados estão voltados tanto para subsidiar intervenções como para impulsionar o desenvolvimento teórico da área.

Palavras-chave: Alimento Funcional. Revisão. Revistas Eletrônicas. 


\section{Abstract}

The Brazilian scientific literature on functional foods is the subject matter of this article. It is a survey, using bibliometric techniques, of articles published on the subject from 2007-2013, available on the Capes Journal Portal. Of the 164 articles in English found in that portal, 22 were selected for analysis. On the subject in question, eight journal articles were found, and two concentrated $63.63 \%$ of production: Food and Nutrition (36.36\%) and Food Science and Technology (27.27\%). The topics covered were parallel antioxidants, fatty acids, prebiotics and probiotics. Unicamp was the institution with the highest number of articles analyzed, representing $13.64 \%$ of the sample. As for the method of approach, there is clear predominance of the quantitative method (54.54\%), which can be explained by the use of sensory and physicochemical reviews, commonly used instruments for quantitative analysis found in $45.45 \%$ of the surveyed items. Most authors have training in nutrition and are tied to universities. The research was exploratory with the use of document analysis, at first, and descriptive, with quantitative method, the second time. The analysis considered the number of articles, higher education institutions, themes, methodology, data collection instruments and training area of the authors. Published works are aimed both to support interventions for boosting the theoretical development of the area.

Keywords: Functional Food. Review. Eletronic Journals.

\section{Introdução}

O princípio de Hipócrates - "deixe o alimento ser teu remédio e o remédio ser teu alimento" -, proferido há 2.500 anos, já demonstrava que os efeitos benéficos de determinados tipos de alimentos sobre a saúde são conhecidos há muito tempo. ${ }^{1}$

A saúde e a qualidade de vida constituem, hoje, uma das preocupações mais salientes de alguns segmentos de consumidores. Deve-se esta preocupação basicamente a três fatores: uma alimentação incorreta, o sedentarismo e o estresse, que originam problemas de saúde como a obesidade; o avanço do conhecimento no campo da saúde, que permite compreender os malefícios desse estilo de vida a médio e longo prazos; e devido à velocidade da difusão do conhecimento, assistimos a uma maior informação entre os consumidores e, desta forma, maior preocupação com a saúde e a qualidade de vida. ${ }^{2}$ 
A ideia de saúde como qualidade de vida condicionada por vários fatores, como paz, abrigo, alimentação, renda, educação, recursos econômicos, ecossistema estável, recursos sustentáveis, equidade e justiça social, surgiu com a Conferência Internacional sobre a Promoção da Saúde, em Ottawa, em 1986. ${ }^{3}$ A Carta de Ottawa ${ }^{4}$ considera a saúde como um conceito positivo, para o qual se fazem necessários recursos pessoais, sociais e capacidade física.

Nesse cenário, vem sendo crescente o interesse pelos chamados "alimentos funcionais", que têm como objetivo contribuir para a promoção da saúde. ${ }^{5,6}$ Pesquisa realizada pela Health Focus em 30 países revelou que 44\% dos consumidores brasileiros das classes A e B escolhem alimentos influenciados pela relação que têm com a saúde - constituindo um dos maiores índices da América Latina. ${ }^{7}$

Vários são os fatores que vêm estimulando o desenvolvimento de alimentos funcionais ao longo dos últimos anos, dentre os quais se destacam: o aumento da expectativa de vida em países desenvolvidos (cujas populações necessitarão de cuidados hospitalares por maior período de tempo), o elevado custo dos serviços de saúde, os avanços na tecnologia de alimentos e ingredientes, a necessidade que as instituições públicas de pesquisa têm de divulgar os resultados de suas investigações e a maior cobertura dos diferentes tipos de mídia dada a essas descobertas e às questões de saúde. ${ }^{8}$

Dado o papel central da investigação científica diante dos desafios que a área enfrenta, o propósito deste estudo é levantar informações acerca da produção científica contemporânea sobre alimentos funcionais, publicadas especificamente em periódicos nacionais na área de Nutrição no período entre 2007 a 2013. Desta forma, o objetivo geral do estudo se volta para a análise da produção científica brasileira a respeito dos alimentos funcionais.

Foram utilizadas as publicações disponíveis no Portal de Periódicos da Capes. Nesse sentido, o presente estudo propõe-se a identificar, por meio do uso de técnicas bibliométricas, as instituições de ensino superior (IES) com maior produção, quais eram os mais produtivos; os temas paralelos a alimentos funcionais tratados nos artigos; os periódicos com maior produtividade sobre o tema; os métodos de pesquisa utilizados; os instrumentos de coleta de dados adotados; os alimentos mais pesquisados. Por fim, foram examinadas as áreas de formação do primeiro autor por meio de consulta ao currículo Lattes.

Justifica-se a escolha do Portal de Periódicos da Capes por ser este um sistema de indexação, pesquisa e disponibilização gratuita da produção científica que busca promover o acesso, organização, disseminação e análise da produção científica de distintas áreas do conhecimento. Assim, este estudo busca contribuir para a pesquisa sobre alimentos funcionais, analisando uma parte relevante da produção científica brasileira. 
$\mathrm{O}$ artigo foi estruturado em quatro partes. Inicialmente, apresentou-se uma visão geral sobre as técnicas bibliométricas e seu uso no estudo da produção científica de uma área, para em seguida, desenvolver o tema central, que são os alimentos funcionais. Posteriormente, delineou-se a metodologia empregada no estudo e em seguida descreveu-se o resultado obtido nesta pesquisa. Por fim, foram apresentadas as conclusões e as recomendações do trabalho realizado.

\section{A Bibliometria na análise da produção científica}

Os alimentos funcionais vêm despertando o interesse de muitos pesquisadores, o que os leva a estudarem esta temática de modo contínuo e sistemático. Um dos caminhos a ser adotado na busca de se compreender a temática é o da bibliometria, que se refere a uma "técnica quantitativa e estatística de medição dos índices de produção e disseminação do conhecimento científico". 9

Destacam-se como principais leis da bibliometria a Lei de Lotka, ${ }^{9}$ voltada para o estudo da produtividade científica dos autores; a Lei de Bradford, voltada para a análise da produtividade de periódicos; e a Lei de Zipf, ${ }^{9}$ que tem como foco a identificação da frequência de palavras no estudo. Pelo uso das técnicas bibliométricas, torna-se possível a medição da produtividade e da dispersão do conhecimento da produção científica e a identificação do modelo de distribuição e frequência de palavras em um texto.

A Lei de Lotka, ${ }^{9}$ também conhecida como Lei do Quadrado Inverso, foi formulada em 1926 e significa que uma larga produção científica se origina de um pequeno número de autores. ${ }^{7} \mathrm{~A}$ Lei de Zipf, ${ }^{9}$ também conhecida como Lei do Menor Esforço, preocupa-se em aferir a frequência com que determinadas palavras aparecem na literatura estudada.

Bradford $^{10}$ fez suas primeiras observações sobre a dispersão de artigos em 1934, mas somente em 1948 elas foram reconhecidas com o status de lei. Assim dispõe o enunciado da lei:

[...] se os periódicos forem ordenados em ordem de produtividade decrescente de artigos sobre um determinado assunto, poderão ser distribuídos num núcleo de periódicos mais particularmente devotados a esse assunto e em diversos grupos ou zonas contendo o mesmo número de artigos que o núcleo, sempre que o número de periódicos e das zonas sucessivas for igual a 1:n:n2.10

\section{Sobre a bibliometria, Bernardino \& Cavalcante afirmam:}

Bibliometria é o estudo dos aspectos quantitativos da produção, disseminação e uso da informação registrada; desenvolve padrões e modelos matemáticos para medir esses processos, usando seus resultados para elaborar previsões e apoiar tomadas de decisão. ${ }^{11}$ 
Desta forma, é possível pela bibliometria delinear um cenário seguro da produção científica sobre alimentos funcionais no país, capaz de nortear pesquisadores e editoras, entre outros, sobre temas a merecerem investimentos.

O uso de técnicas bibliométricas permite analisar o desenvolvimento e a distribuição da produção científica, considerando, ao mesmo tempo, a estrutura social dos que realizam essa produção e a interação existente entre os que produzem e os que consomem este conteúdo. ${ }^{10}$ O estudo bibliométrico objetiva medir e quantificar os resultados para minimizar as possíveis distorções quando da análise e interpretação dos dados. ${ }^{12}$ Por esse estudo, tem-se o uso de técnicas estatísticas e matemáticas para objetivamente analisar a produção científica.

\section{Alimentos funcionais}

Na década de 1980, foi introduzido no Japão o termo "alimentos funcionais" ( foshu - foods for specified health use), para caracterizar uma nova concepção de alimentos que integram propriedades medicinais, para diminuir os custos com saúde. Esse termo foi lançado como parte de um programa de governo, cujo objetivo era desenvolver alimentos saudáveis para uma população que envelhecia e apresentava alta expectativa de vida. ${ }^{1}$

Os alimentos funcionais são aqueles que, além de contribuir com a nutrição, contêm substâncias que podem ser consideradas biologicamente ativas, produtoras de benefícios clínicos ou de saúde. São capazes de reduzir o risco de algumas doenças e auxiliar em funções fisiológicas do organismo. ${ }^{13}$

Outra definição aceita descreve os alimentos funcionais como

[...] alimentos para os quais pode ser satisfatoriamente demonstrado que eles afetam beneficamente uma ou mais funções do organismo, além de garantirem efeitos nutricionais adequados, conduzindo a uma melhoria do estado geral de saúde e bem-estar elou a uma redução do risco de doenças. ${ }^{14-16}$

Em relação à definição, é importante ressaltar a necessária distinção em relação ao termo "nutracêuticos". Enquanto os alimentos funcionais se referem a alimentos ou bebidas consumidos na alimentação cotidiana que podem trazer benefícios à saúde devido a fatores fisiologicamente saudáveis, os nutracêuticos se relacionam a uma ampla gama de alimentos e componentes alimentares com forte apelo à saúde e abrangem suplementos alimentares, nutrientes isolados, produtos herbais ou alimentos processados. ${ }^{1}$ Segundo Campos,

[...] sob um ponto de vista prático, um alimento funcional pode ser um alimento natural; um alimento ao qual foi adicionado um componente, ou um alimento do qual foi removido um componente através de processos tecnológicos ou biotecnológicos. ${ }^{17}$ 
A legislação brasileira não define claramente os alimentos funcionais, mas alega a propriedade funcional como sendo "aquela relativa ao papel metabólico ou fisiológico que o nutriente ou não nutriente tem no crescimento, desenvolvimento, manutenção e outras funções normais do organismo humano". ${ }^{18}$ Além disso, o Ministério da Saúde, através da Agência Nacional de Vigilância Sanitária (ANVISA), regulamentou os alimentos funcionais através das resoluções ANVISA/MS 16/99; ${ }^{19}$ ANVISA/MS 17/99:;0 ANVISA/MS 19/99. ${ }^{21}$

Esses alimentos possuem potencial para promover a saúde através de mecanismos não previstos na nutrição convencional, devendo ser salientado que esse efeito restringe-se à promoção da saúde e não à cura de doenças. ${ }^{22} \mathrm{~A}$ incorporação da dieta a um estilo de vida saudável, uma vez que esta pode estar associada à prevenção de doenças e promoção da saúde, levou à criação de um mercado para esses produtos. ${ }^{8}$

Um alimento funcional pode ser classificado de acordo com o alimento em si ou conforme os componentes bioativos nele presentes, como, por exemplo, os probióticos, as fibras, os fitoquímicos, as vitaminas, os minerais, as ervas, os ácidos graxos ômega 3 ( $\omega-3)$, além de determinados peptídeos e proteínas. ${ }^{8}$ Existem outras duas formas de classificação desses alimentos: a) quanto à fonte, podendo ser de origem vegetal ou animal; b) quantos aos benefícios que oferecem, atuando em seis áreas do organismo, sendo elas: no sistema gastrointestinal; no sistema cardiovascular; no metabolismo de substratos; no crescimento, no desenvolvimento e diferenciação celular; no comportamento das funções fisiológicas e como antioxidantes. ${ }^{23}$

Os alimentos funcionais são a nova tendência do poderoso mercado alimentício neste início do século XXI. ${ }^{24}$ Entre os fatores-chave que explicam o êxito dos alimentos funcionais, a preocupação crescente pela saúde e pelo bem-estar, mudanças na regulamentação dos alimentos e a crescente comprovação científica das relações existentes entre dieta e saúde. ${ }^{25}$

O desenvolvimento de alimentos funcionais é um campo que está repleto de oportunidades. O consumidor está exigindo alimentos que suprem suas necessidades nutricionais, e que possam contribuir para o fortalecimento da sua saúde e manutenção do seu bem-estar, além de retardar o máximo possível o aparecimento de doenças.

O mercado mundial de alimentos funcionais, sobretudo nos países desenvolvidos, mostrou expressivo crescimento na década de 1990. Devemos salientar que, como qualquer outro mercado, o de alimentos funcionais é influenciado por diversos atores. O campo econômico é um campo de ação socialmente construído em que se enfrentam agentes dotados de recursos diferentes em função do volume e da estrutura do capital que possuem, sob suas diferentes formas: financeiro, cultural, tecnológico, jurídico, organizacional, comercial e simbólico e, em função desses recursos, os atores definem estratégias de ação no âmbito dos limites impostos pela estrutura do campo, em particular pelo seu grau de concentração. ${ }^{26,27}$ 
O Estado exerce grande importância no processo de construção social do mercado. Dentre todas as características das sociedades nas quais a ordem econômica está "imersa”, as mais importantes para as sociedades contemporâneas são a forma e a força de sua tradição estadista. ${ }^{28} \mathrm{O}$ Estado pode influenciar na construção da demanda por meio da produção dos sistemas de preferências individuais e da atribuição dos recursos necessários (por exemplo, orientação do crédito, ajudas fiscais etc. ${ }^{28}$

\section{Metodologia}

Os dados do presente estudo consistiram em artigos publicados em periódicos nacionais entre 2007 a 2013, disponíveis no Portal de Periódicos da Capes em janeiro de 2014. A inclusão das publicações sobre alimentos funcionais foi definida a partir da análise dos títulos, resumos e palavras-chave dos que continham o tema "alimentos funcionais" nos artigos publicados em periódicos nacionais. Para a análise, foram utilizados apenas artigos.

Este estudo pode ser caracterizado como exploratório, com método quantitativo. A pesquisa é do tipo documental, ${ }^{29-32}$ visto que o material objeto de estudo são artigos publicados em revistas científicas, um tipo de publicação periódica. Em um segundo momento, a pesquisa assume caráter descritivo.

Como o objetivo geral do estudo foi analisar a produção sobre alimentos funcionais no Brasil, na etapa exploratória e quantitativa da pesquisa realizou-se levantamento dos artigos científicos publicados em periódicos nacionais e disponibilizados no Portal de Periódicos da Capes. Para tanto, optou-se por analisar os artigos produzidos entre 2007 e 2013, visto que esse período se refere à produção mais atualizada sobre o tema.

Num primeiro momento da pesquisa, buscou-se qualificar todos os artigos científicos sobre alimentos funcionais, disponibilizados no portal. Assim, procedeu-se à filtragem, por busca de artigos sobre o tema em questão, utilizando os seguintes critérios: periódicos avaliados por pares, artigos que apresentavam a expressão "alimentos funcionais" no título, resumo e/ou nas palavraschave, idioma: português e entre os anos de 2007 e 2013.

Após esta abordagem, os artigos foram separados para análise. Nessas condições, encontrou-se um total de 22 artigos científicos, que passaram a integrar a segunda etapa da pesquisa, descritiva quantitativa. Nesta etapa, a finalidade inicial foi identificar, nos artigos em estudo, as seguintes variáveis: as IES com maior produção, quais eram os mais produtivos; os temas paralelos a alimentos funcionais tratados nos artigos; os periódicos com maior produtividade sobre o tema; 
os métodos de pesquisa utilizados; os instrumentos de coleta de dados adotados; alimentos mais pesquisados e; por fim, foram examinadas as áreas de formação do primeiro autor por meio de consulta ao currículo Lattes.

Em seguida, foi realizada a quantificação dos resultados, com a contagem simples de frequência. Ao concluir esta etapa, em cada um dos artigos avaliados, os dados foram analisados por meio de estatísticas descritivas, utilizando-se como apoio o software Microsoft Excel. Os resultados foram reunidos em tabelas e gráfico.

\section{Resultados e discussões}

\section{Perfil da amostra}

\section{Autores dos artigos e IES ligadas a pesquisas sobre alimentos funcionais}

Nos artigos analisados, não foi constatada a presença de um mesmo autor em mais de um artigo. Isso pode inferir a falta de referências no meio acadêmico quando o assunto trata de alimentos funcionais, assim como pode estar diretamente ligado ao fato de a pesquisa sobre esses alimentos ser ainda recente no país.

Verificiou-se que algumas IES apresentam participação maior na produção científica sobre alimentos funcionais. Conforme pode ser observado na tabela 1, UNICAMP, UFRJ e USP têm juntas uma representatividade de $31,82 \%$ dos artigos pesquisados.

Tabela 1. Distribuição das produções científicas por IES. Brasil, 2007-2013.

\begin{tabular}{cccc}
\hline IES & $\mathrm{f}($ TOTAL $)$ & $\mathrm{f}(\%)$ \\
\hline UNICAMP & 3 & $13,64 \%$ \\
UFRJ & 2 & $9,09 \%$ \\
USP & 2 & $9,09 \%$ \\
Outros & 15 & $68,18 \%$ \\
\hline
\end{tabular}

Fonte: Resultados da pesquisa 


\section{Assuntos mais frequentes relacionados ao tema}

Quanto aos assuntos relacionados a alimentos funcionais, observou-se que antioxidantes, ácidos graxos, prebióticos e probióticos são alguns dos assuntos paralelamente tratados quando se fala em alimentos funcionais. Isso foi observado em alguns artigos. Por exemplo, tópicos sobre antioxidantes foram encontrados em três artigos (13,64\% do total); já tópicos sobre prebióticos, em cinco (22,73\% do total). Prebióticos são componentes alimentares não digeríveis pelo organismo que atuam proporcionando um substrato de crescimento de microorganismos dos intestinos, mudando, assim, a atividade da flora intestinal. ${ }^{1}$

Em relação à saúde, contribuem para a modulação de funções fisiológicas importantes - como absorção de cálcio, metabolismo lipídico - e modulação da composição da microbiota intestinal, exercendo um papel fundamental na fisiologia intestinal e na redução do risco de câncer de cólon. ${ }^{33}$ O uso de prebióticos em alimentos pode aumentar a crocância em snacks e cereais e melhorar a durabilidade de pães e bolos. Sua solubilidade permite que sejam incorporados em alimentos líquidos ou bebidas, sem alterar as características sensoriais dos alimentos. ${ }^{34}$

\section{Distribuição de artigos por periódicos}

A partir do levantamento realizado, pode-se constatar que alguns periódicos apresentam volume maior de publicações sobre alimentos funcionais. De oito periódicos analisados, dois concentram 63,63\% da produção, ou seja, mais da metade dos artigos analisados. Ao verificar a distribuição do universo das publicações sobre alimentos funcionais, verifica-se que o periódico Alimentos e Nutrição possui o maior número de artigos sobre a temática pesquisada, representando 36,36\% do total, seguido pelo periódico Ciência e Tecnologia de Alimentos, que concentra 27,27\% do total. A escolha dos periódicos relaciona-se com a área de formação dos autores (em sua maioria da área de Nutrição e Engenharia de Alimentos), além de revelar diferenças nas especificidades temáticas e escopo dessas publicações.

A distribuição das publicações sobre alimentos funcionais por periódico (tabela 2) também revela diferenças entre os veículos de divulgação quanto à presença de estudos sobre o tema. Tais diferenças podem ser atribuídas a diversos fatores, dentre os quais a preferência dos autores por determinados periódicos, a especificidade temática de alguns destes periódicos, assim como a periodicidade dos mesmos. 
Tabela 2. Distribuição de artigos por periódicos. Brasil, 2007-2013.

\begin{tabular}{ccc}
\hline PERIÓDICOS & $\mathrm{f}(\mathrm{TOTAL})$ & $\mathrm{f}(\%)$ \\
\hline Alimentos e Nutrição & 8 & $36,36 \%$ \\
Ciência e Tecnologia de Alimentos & 6 & $27,27 \%$ \\
Revista de Nutrição & 2 & $9,09 \%$ \\
Ciência Rural & 2 & $9,09 \%$ \\
Outros & 4 & $18,20 \%$ \\
\hline
\end{tabular}

Fonte: Resultados da pesquisa

\section{Metodologias utilizadas}

Quanto aos métodos de pesquisa utilizados nos estudos publicados nos artigos analisados, os do tipo misto são a preferência da maioria dos autores (tabela 3). Este fato pode ser explicado, pois na maioria dos artigos o intuito dos autores era obter dados sobre os compostos presentes nos alimentos estudados e a percepção sobre o consumo de tais alimentos.

O levantamento demonstrou que os estudos quantitativos representam a maioria com $54,54 \%$ de participação, seguidos pelos estudos qualitativos, que estão presentes em 36,36\% dos artigos. Tendo em vista que as metodologias possuem estreita relação com os objetivos da pesquisa, foi possível identificar como principais focos da pesquisa: obter dados sobre os compostos presentes nos alimentos estudados e analisar a percepção sobre o consumo de tais alimentos. Segundo pesquisas relacionadas à alimentação e à Nutrição, as metodologias qualitativas ampliaram de forma significativa a interlocução dessas áreas com as Ciências Sociais e Humanas. Destaca-se que estudos qualitativos representam um avanço, embora requeiram aperfeiçoamento teóricometodológico para superar os estudos descritivos. ${ }^{35}$ 
Tabela 3. Distribuição de metodologia empregada nos artigos. Brasil, 2007-2013.

\begin{tabular}{ccc}
\hline MÉTODOS DE PESQUISA & $\mathrm{f}(\mathrm{TOTAL})$ & $\mathrm{f}(\%)$ \\
\hline Quantitativos & 12 & $54,54 \%$ \\
Qualitativos & 8 & $36,36 \%$ \\
Mistos & 2 & $9,09 \%$ \\
\hline
\end{tabular}

Fonte: Resultados da pesquisa

\section{Instrumentos de coleta de dados}

$\mathrm{Na}$ continuidade do levantamento dos artigos publicados sobre alimentos funcionais em periódicos nacionais, analisaram-se os instrumentos de coleta de dados adotados pelos autores. Primeiramente, é importante ressaltar a estreita relação entre objetivos, metodologias de pesquisa e instrumentos de coletas de dados.

Considerando o exposto em relação às metodologias, percebeu-se que a análise sensorial, análise físico-química e os questionários são os instrumentos preferidos pelos pesquisadores (tabela 4). Segundo a Associação Brasileira de Normas Técnicas, a análise sensorial é uma disciplina científica usada para interpretar reações das características dos alimentos e materiais como são percebidas pelos sentidos. ${ }^{36}$ Essa metodologia é amplamente utilizada pelo setor de alimentos, como forma de analisar a qualidade e aceitabilidade de um produto. ${ }^{37}$ Essa metodologia, em geral, é realizada por uma equipe cujo objetivo é analisar as características sensoriais de um produto. ${ }^{37}$ Como resultado, é possível avaliar, por exemplo, a matéria-prima a ser utilizada em um novo produto, o efeito de processamento, a qualidade da textura, o sabor, a estabilidade de armazenamento, a reação do consumidor, entre outros. Já as análises físico-químicas baseiam-se em técnicas instrumentais que buscam quantificar os analitos, mesmo em concentrações muito baixas. Essas técnicas são bastante úteis, principalmente em função dos avanços tecnológicos na ciência dos alimentos, para desenvolver estudos toxicológicos, de identidade e de qualidade de alimentos. ${ }^{38}$

Em síntese, como a maioria dos artigos possuía caráter quantitativo e exploratório, há uma preferência por instrumentos que meçam os constituintes dos alimentos em estudo e percepção do seu consumo, o que explica, por exemplo, a predominância da análise sensorial nos artigos pesquisados. 
Tabela 4. Distribuição de instrumentos de coletas de dados utilizados nos artigos. Brasil, $2007-2013$.

\begin{tabular}{ccc}
\hline INSTRUMENTOS & $\mathrm{f}(\mathrm{TOTAL})$ & $\mathrm{f}(\%)$ \\
\hline Análise Sensorial & 6 & $27,27 \%$ \\
Análise Físico-Química & 4 & $18,18 \%$ \\
Questionários & 3 & $13,64 \%$ \\
Avaliação de Propriedades Reológicas & 2 & $9,09 \%$ \\
Pesquisa Documental & 2 & $9,09 \%$ \\
Pesquisa Bibliográfica & 2 & $9,09 \%$ \\
Observação & 1 & $4,55 \%$ \\
Outros & 7 & $31,82 \%$ \\
\hline
\end{tabular}

Fonte: Resultados da pesquisa

\section{Alimentos pesquisados}

Pesquisar a soja foi a preferência da maioria dos autores. A soja, alimento de consumo universal, é de grande importância no que se refere a alimentos funcionais, por ter grande qualidade de proteína, podendo ser utilizada de forma preventiva e terapêutica no tratamento de doenças cardiovasculares, câncer, osteoporose e sintomas da menopausa. ${ }^{39}$

Com relação ao conceito de alimento funcional, a soja abre perspectivas às indústrias alimentícias para o desenvolvimento de diversas formulações alimentares, o que ajuda a justificar sua preferência dentre os pesquisadores. Além disso, o grande investimento na produção brasileira e o investimento em transgênicos colaboram com esse indicativo. O consumo da soja in natura ou mesmo processada na forma de derivados tem despertado grande interesse da população, sobretudo dos pesquisadores, não só por ser considerada fonte importante de nutrientes de baixo custo, mas particularmente por sua capacidade de diminuir o risco de doenças crônico-degenerativas. ${ }^{40}$

Apesar de o consumo de soja ter aumentado de forma significativa e aparecer recorrentemente ligado a benefícios à saúde, existem também controvérsias a esse respeito, pouco conhecidas. Discute-se ainda o caráter socioambiental dos riscos relacionados à sojicultura, frequentemente ignorado na definição de soja como alimento saudável e seguro. ${ }^{41} \mathrm{O}$ uso de sementes transgênicas, por exemplo, apresenta repercussões negativas sobre o habitat e a saúde e qualidade de vida dos seres humanos. ${ }^{42}$ 


\section{Formação dos autores}

No que diz respeito à área de formação superior inicial (primeira titulação em nível superior) do primeiro autor, obtida através da consulta ao currículo Lattes, observou-se que a maioria dos autores dos artigos analisados são bacharéis em Nutrição, representando 27,27\% do total, como se pode verificar na figura 1 . Além disso, constatou-se que grande parte deles são professores ou estão vinculados a instituições de ensino superior.

Devido às mudanças no padrão alimentar atual e seus efeitos na saúde, acarretando alto índice de doenças crônicas e/ou degenerativas, o campo da nutrição vem se dedicando crescentemente à relação entre dieta e saúde. ${ }^{43} \mathrm{~A}$ autora aponta que:

[...] este novo conceito de alimentos promotores de boa saúde está emergindo como uma nova fronteira no desafio dos profissionais de nutrição e introduzindo a necessidade dos nutrientes tradicionais, como estabelecido ao longo de todos os anos de estudos da Nutrição, mas muito ampliada para o conceito nutrientes preventivos. ${ }^{43}$

Outra área de grande destaque na produção acadêmica sobre alimentos funcionais foi a Engenharia de Alimentos, representando 22,73\% do total. Na CAPES, esse campo se encontra dentro da área de avaliação "Ciência do Alimento". Como foco, esta se ocupa em dominar todo o processo de fabricação, conservação, armazenamento e transporte de alimentos industrializados. Assim, o interesse da área pelo tema se deve ao grande investimento da indústria alimentícia em alimentos funcionais.

Para Raud, "os alimentos funcionais são a nova tendência do poderoso mercado alimentício neste início do século XXI”, de modo que nas gôndolas dos supermercados se pode observar uma profusão de novos produtos que prometem contribuir na busca por uma vida mais saudável. ${ }^{2} \mathrm{~A}$ demanda por produtos dessa natureza vem impulsionando a pesquisa para desenvolver produtos tais como iogurtes, margarinas, leites fermentados, cereais, águas minerais e afins - que prometem contribuir na prevenção de doenças como as cardiovasculares, alergias, problemas intestinais etc.

O relatório Brasil Foods Trends $2020^{44}$ identifica, entre as tendências de "saudabilidade e bem-estar", o crescimento da procura por alimentos funcionais, como consequência da maior preocupação dos consumidores com a nutrição. O documento destaca que o consumo de produtos funcionais tem formado diferentes nichos de mercado, como, por exemplo, os de produtos benéficos ao desempenho físico e mental, para a saúde cardiovascular, saúde gastrointestinal, para melhorar o estado de ânimo (energéticos) e para relaxar, entre outros. ${ }^{44}$

Outro dado importante e já esperado: a maioria dos autores é proveniente de instituições públicas, visto que a pesquisa sobre o tema em questão ainda se concentra no ambiente universitário. 


\section{Área de Formação dos Autores}

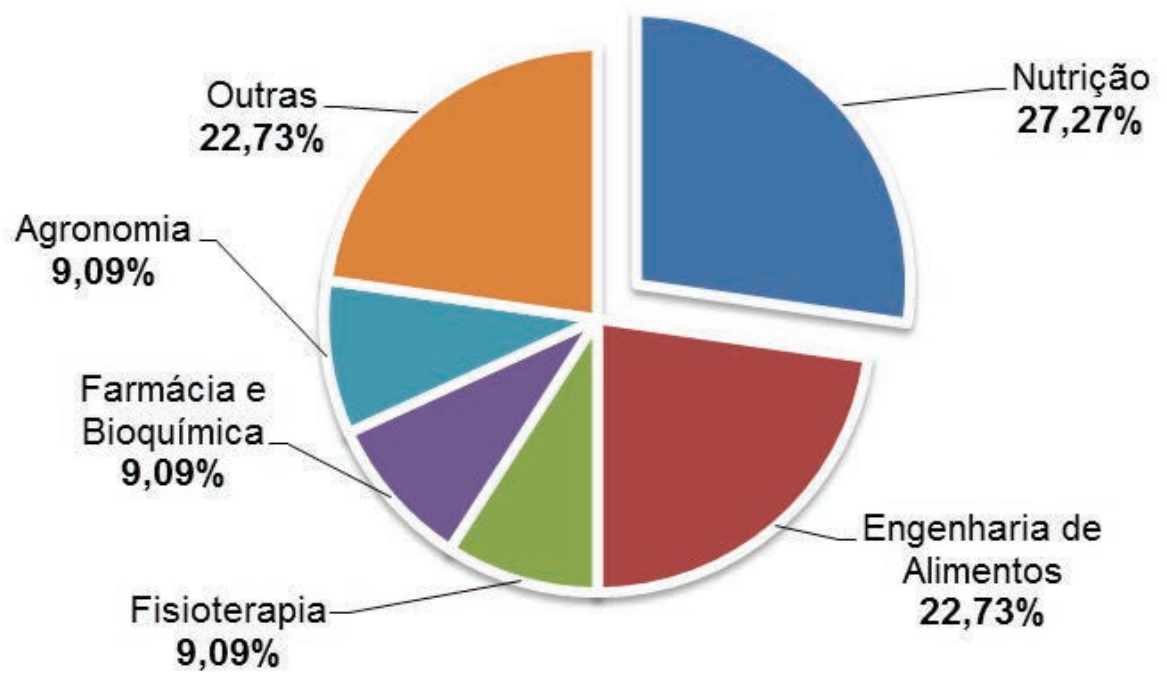

Figura 1. Gráfico da distribuição das áreas de formação dos autores. Brasil, 2007-2013.

Fonte: Resultados da pesquisa

\section{Conclusões}

Considerando que o levantamento realizado oferece importantes contribuições para uma maior caracterização da produção científica nacional sobre alimentos funcionais, podemos perceber, pelos dados e pela análise feita, que existe um número baixo de publicações em periódicos brasileiros sobre alimentos funcionais.

Analisando-se a produção científica publicada em periódicos nacionais, é possível observar algumas tendências: há predominância de pesquisas quantitativas e são menos frequentes as investigações que utilizam uma abordagem mista (qualitativa e quantitativa) para tratar o tema.

Os resultados evidenciam uma diversidade temática e metodológica da produção científica sobre alimentos funcionais, bem como a preocupação dos autores em abordar temas correlacionados, como antioxidantes, ácidos graxos, probióticos e prebióticos. Esta realidade demonstra que os trabalhos sobre alimentos funcionais não estão voltados apenas para a reprodução de assuntos, mas que estão considerando a diversidade de assuntos relacionados ao tema. 
Outro ponto importante é que o levantamento indica que a produção de conhecimento está voltada tanto para a execução de intervenções, desenvolvimento de produtos alimentícios funcionais, como para impulsionar o desenvolvimento teórico sobre o tema. Isto ficou evidente ao se observar que os estudos estavam voltados tanto para o desenvolvimento como para as revisões temáticas sobre os mesmos.

Algo que poderia aprofundar o estudo na área seria o desenvolvimento de pesquisas que contemplassem períodos mais longos, no intuito de identificar tendências e mudanças temáticas da produção científica sobre alimentos funcionais, assim como o estudo em outros tipos de alimentos. Como verificado na presente pesquisa, os estudos sobre alimentos funcionais não apresentam variedade muito grande, concentrando-se em alimentos como a soja.

Outro aspecto que poderia contribuir para esta análise seria a inclusão de dissertações e teses sobre o tema, por constituírem importantes fontes de produção científica.

\section{Referências}

1. Moraes FP, Colla LM. Alimentos funcionais e nutracêuticos: definições, legislação e benefícios à saúde. Revista Eletrônica de Farmácia 2006; 3(2):109-122.

2. Oliveira HS, Cardoso PR. O marketing dos alimentos funcionais: reflexões sobre o mercado e os consumidores. Biblioteca on-Line de Ciências da Comunicação [Internet] 2010; 9 f. Disponível em: http://www.bocc.ubi.pt/pag/bocc-cardoso-marketing.pdf

3. Backes MTS. Conceitos de saúde e doença ao longo da história sob o olhar epidemiológico e antropológico. Rev. Enferm. UERJ 2009; 17(1):111-117.

4. World Health Organization. The Ottawa charter [Internet]. Disponível em: http://www.who.int/ healthpromotion/conferences/previous/ottawa/en/

5. Granato D, Branco GF, Nazzaro F, Cruz AG, Fria JAF. Functional foods and nondairy probiotic food development: trends, concepts, and products. Comprehensive Reviews in Foods Science and Food Safety 2010; 9(3):292-301.

6. Annunziata A, Vecchio R. Functional foods development in the European market: a consumer perspective. Journal of Functional Foods 2011; 3(3):223-228.

7. Oliveira D, Fernandes D. Revolução na mesa. Isto É Dinheiro [Internet] 2004. Disponível em: http:// www.istoedinheiro.com.br/noticias/negocios/20040121/revolucao-mesa/15984.shtml

8. Arvanitoyannis IS, Houwelingen-koukaliaroglou MV. Functional foods: a survey of health, claims, pros and cons, and current legislation. Crit. Rev. Food Sci. Nutr. 2005; 45(5):385-404.

9. Araújo CA. Bibliometria: evolução histórica e questões atuais. Em Questão 2006; 12(1):11-32.

10. Pinheiro, LVR. Lei de Bradford: uma reformulação conceitual. Ciência da Informação 1983; 12(2):59-80. 
11. Bernardino MCR, Cavalcante RS. Análise de citações dos artigos da revista Ciência da Informação no período de 2000-2009. Em Questão 2011; 17(1):247-263.

12. Gnecco Júnior, L, Ramos VL, Kich JIF, Bernardini, IS, Gnecco, INS. Análise bibliométrica da produção científica nos Colóquios I a IX. Anais X Colóquio Internacional sobre Gestão Universitária na América do Sul; 12 ago. 2010;. Florianópolis, SC. Florianópolis: UFSC; 2010. p. 1-16.

13. Goldberg I. Functional foods. New York: Chapman and Hall; 1994. p. 183-201.

14. Stanton C, Fitzgerald G, Ross P, Desmond C, Coakley M Collins K. Challenges facing development of probiotic-containing functional foods. In: Farnworth ER editor. Handbook of fermented functional foods. Boca Raton: CRC Press; 2003. p. 27-58.

15. Roberfroid MB. Prebiotics: the concept revisited. J. Nutr. 2007; 137(3):830-837.

16. European Commission Concerted Action On Functional Food Science In Europe. Scientific concepts of functional foods in Europe consensus document. Br. J. Nutr. 1999; 81(4):1-27.

17. Fé CMC. Impacto da intervenção educativa no consumo de alimentos funcionais por usuários de restaurantes self services [Dissertação]. Teresina: Universidade Federal do Piauí, 2010.

18. Brasil. Resolução n. 18, de 30 de abril de 1999. Aprova o Regulamento Técnico que Estabelece as Diretrizes Básicas para Análise e Comprovação de Propriedades Funcionais e ou de Saúde Alegadas em Rotulagem de Alimentos. Diário Oficial da União 03 maio 1999.

19. Brasil. Resolução n. 16, de 30 de abril de 1999. Aprova o Regulamento Técnico de Procedimentos para Registro de Alimentos e ou Novos Ingredientes. Diário Oficial da União 03 maio 1999.

20. Brasil. Resolução n. 17, de 30 de abril de 1999. Aprova o Regulamento Técnico que Estabelece as Diretrizes Básicas para Avaliação de Risco e Segurança dos Alimentos. Diário Oficial da União 03 maio 1999.

21. Brasil. Resolução n. 19, de 30 de abril de 1999. Aprova o Regulamento Técnico de Procedimentos para Registro de Alimento com Alegação de Propriedades Funcionais e ou de Saúde em sua Rotulagem. Diário Oficial da União 3 de maio de 1999.

22. Sanders ME. Probiotics: considerations for human health. Nutr. Rev 2003; 61(3):91-99.

23. Souza PHM, Souza Neto MH, Maia GA. Componentes funcionais nos alimentos. Boletim da SBCTA 2003; 37(2):127-135.

24. Heasman M, Mellentin J. The functional foods revolution. healthy people, healthy profits? London: Earthscan; 2001.

25. Hasler CM. The changing face of functional foods. J. Am. Coll. Nutr 2000; 19(Supl. 5):499S-506S.

26. Brochier P. O campo econômico. Política \& Sociedade 2005; 4(6):15-57.

27. Bourdieu P. Le champ économique. Actes de la Recherche en Sciences Sociales 1997; 119(119):48-66.

28. Brochier P. Les structures sociales de l'économie. Paris: Seuil; 2000.

29. Gil AC. Como elaborar projeto de pesquisa. São Paulo: Atlas; 2002. 
30. Gil AC. Métodos e técnicas de pesquisa social. São Paulo: Atlas; 2007.

31. Severino AJ. Metodologia do trabalho científico. São Paulo: Cortez; 2002.

32. Pimentel A. O método da análise documental: seu uso numa pesquisa histórica. Cadernos de Pesquisa 2001; 114:179-195.

33. Raud C. Os alimentos funcionais: a nova fronteira da indústria alimentar análise das estratégias da Danone e da Nestlé no mercado brasileiro de iogurtes. Rev. Sociol. Polít. 2008; 16(31):85-100.

34. Al-Sheraji SH, Ismail A, Manap MY, Mustafa S, Yusof RM, Hassan FA. Prebiotics as functional foods: a review. Journal of Functional Foods 2013; 5(4):1542-1553.

35. Canesqui AM. Pesquisas qualitativas em nutrição e alimentação. Rev. Nutr. 2009; 22(1):125-139.

36. Associação Brasileira de Normas Técnicas. Análise sensorial. Vocabulário. NBR ISO 5492. São Paulo: ABNT; 2014.

37. Teixeira LV. Análise sensorial na Indústria de Alimentos. Rev. Inst. Latic. Cândido Tostes 2009; 366(64):12-21.

38. Shibao J, Santos GFA. Edulcorantes em alimentos: aspectos químicos, tecnológicos e toxicológicos. São Paulo: Phorte; 2009.

39. Behrens JH, Silva MAAP. Atitude do consumidor em relação à soja e produtos derivados. Ciênc. Tecnol. Aliment. 2004; 24(3):431-439.

40. Lof M, Weiderpass E. Epidemiologic evidence suggests that dietary phytoestrogen intake is associated with reduced risk of breast, endometrial, and prostate cancers. Nutr. Res. 2006; (26):609-619.

41. Azevedo E. Riscos e controvérsias na construção social do conceito de alimento saudável: o caso da soja. Rev. Saúde Pública 2011; 45(4):781-788.

42. Plataforma Brasileira de Direitos Humanos Econômicos, Sociais, Culturais e Ambientais. Relatorias Nacionais em Direitos Humanos Econômicos, Sociais, Culturais e Ambientais. Informe 2006. Rio de Janeiro: Plataforma DhESCA Brasil; 2007. Disponível em: http://www.dhnet.org.br/dados/ relatorios/r_dhescas_br/informe_relatores_dhesc_2006.pdf

43. De Angelis R. Novos conceitos em nutrição. Reflexões a respeito do elo dieta e saúde. Arq. Gastroenterol. 2001; 38(4):269-271.

44. Federação das Indústrias do Estado de São Paulo. Brasil Foods Trends 2020. São Paulo: FIESP; 2010. 176 f. Disponível em: URL: http://www.brasilfoodtrends.com.br/Brasil_Food_Trends/index.html

Recebido: $21 / 6 / 2014$

Revisado: 16/9/2014

Aprovado: 04/11/2014 
